\title{
MODELADO CINÉTICO DEL MICOPARASITISMO POR Trichoderma harzianum CONTRA Cladosporium cladosporioides AISLADO DE FRUTOS DE CACAO (Theobroma cacao)
}

KINETIC MODELING OF MYCOPARASITISM BY Trichoderma harzianum AGAINST Cladosporium cladosporioides ISOLATED FROM COCOA FRUITS (Theobroma cacao)

\author{
Teresa Romero-Cortes ${ }^{1}$, Pablo Antonio López-Pérez ${ }^{1}$, Mario Ramírez-Lepe ${ }^{2}$ y Jaime Alioscha \\ Cuervo-Parra $^{1^{*}}$
}

${ }^{1}$ Escuela Superior de Apan, Carretera Apan-Calpulalpan, Km. 8, Chimalpa Tlalayote s/n, Colonia Chimalpa, Apan, Hgo., México. C.P. 43900.

${ }^{2}$ Unidad de Investigación y Desarrollo en Alimentos,2Instituto Tecnológico de Veracruz, Av. Miguel Angel de Quevedo 2779, Col Formando Hogar. C.P. 91897. Veracruz, Ver. México.

* Autor para correspondencia E-mail: jalioscha@gmail.com

\section{RESUMEN}

Cepas de Cladosporium aisladas de frutos de Theobroma cacao e identificadas como C. cladosporioides se confrontaron contra Trichoderma harzianum VSL291 por medio de experimentos de confrontación. El Índice de Biocontrol (IBC) de T. harzianum VSL291 sobre $C$. cladosporioides observado a los siete días fue de $76,29 \%$. Las mayores actividades proteolíticas detectadas en la cepa antagonista con paredes celulares de C. cladosporioides, Colletotrichum gloeosporioides y Corynespora cassicola se asociaron con un aumento de las actividades de las enzimas $\beta-1,3$ glucanasas, quitinasas, lipasas, proteasas y xilanasas. Estos resultados sugieren que las enzimas proteolíticas de acuerdo a su grado de inducción podrían participar en el efecto antagonista de T. harzianum VSL291 contra los hongos confrontados. Por otra parte, los valores más altos del IBC sugieren que las cepas de Trichoderma podrían tener la capacidad para antagonizar de manera más eficiente contra los patógenos fúngicos de este cultivo. Finalmente, se desarrolló un modelo cinético no estructurado bajo un sistema de ecuaciones diferenciales acopladas en función del tiempo basado en el mecanismo del micoparasitismo. Este modelo predice las concentraciones de las actividades enzimáticas de Trichoderma ( $\beta-1,3$ glucanasas, quitinasas, lipasas, proteasas, xilanasas) con las paredes celulares de $C$. cladosporioides. Los parámetros cinéticos se ajustaron a los datos experimentales y se validaron por un coeficiente de correlación.

Palabras clave: micoparasitismo simulado, Theobroma cacao, caracterización molecular, parámetros cinéticos.

\section{ABSTRACT}

Cladosporium strains isolated from Theobroma cacao pods identified as C. cladosporioides were confronted against Trichoderma harzianum VSL291 strain by confrontation experiments. Biocontrol index (IBC) of T. harzianum VSL 291 on C. cladosporioides observed at seven days was $76.29 \%$. The highest proteolytic activities detected in the antagonistic strain with C. cladosporioides, Colletotrichum gloeosporioides and Corynespora cassicola cell walls were associated with increased enzymatic activities of $\beta-1,3$ glucanases, chitinases, lipases and proteases. These results suggest that proteolytic enzymes according to their degree of induction could participate in the antagonistic effect of T. harzianum VSL291 against the fungi tested. Moreover, the highest IBC values obtained suggest that the Trichoderma strains could be capable of antagonizing more efficiently against fungal 
pathogens of these crops. Finally, a kinetic model based on unstructured mycoparasitism mechanism was developed; this model was designed under a system of coupled differential equations with time. This model can predict the concentrations of the enzyme activities of Trichoderma ( $\beta-1,3$ glucanases, chitinases, lipases, proteases, xylanases) in the cell walls of $C$. cladosporioides. Kinetic parameters were fitted to the experimental data and validated by a correlation coefficient.

Key words: simulated mycoparasitism, Theobroma cacao, molecular characterization, kinetic parameters.

\section{INTRODUCCIÓN}

El cacao (Theobroma cacao L.) es un cultivo de gran valor comercial a nivel mundial, cuyo fruto se procesa para ser utilizado en diversos productos. Sin embargo, el gran volumen de residuos que generalmente permanecen dispersos en la plantación y la acumulación de humedad favorecen el crecimiento y la propagación de hongos fitopatógenos. Las enfermedades de mayor impacto en el cultivo son la "moniliasis" causada por Moniliophthora roreri (Cuervo-Parra et al., 2011b), la "escoba de bruja" causada por Moniliophthora perniciosa (Kilarul et al., 2007) y la "mancha negra" causada por varias especies del género Phytophthora (Djocgoue et al., 2006). No obstante, otras enfermedades no específicas del cultivo del cacao como "la roña" o "podredumbre de Cladosporium" causada por varias especies del género Cladosporium, pueden ocasionar pérdidas económicas cuando no son controladas (Riascos et al., 2011). La podredumbre de Cladosporium puede infestar y matar plántulas y ramas, retrasar la producción y floración, y afectar la calidad de los frutos, y aunque el hongo no causa deterioro de la pulpa del grano, la apariencia y aceptación de los frutos en el mercado se ve reducido (Goes, 1998). De las 772 especies que comprende este género, Cladosporium herbarum y C. cladosporioides se encuentran entre las especies más predominantes de los ambientes internos y externos, prevaleciendo en materia orgánica en descomposición, por lo cual, comúnmente se encuentran como contaminantes de alimentos (Schubert et al., 2007). En los últimos años, el género Cladosporium ha sido estudiado morfológica y molecularmente (Braun et al., 2008; Bensch et al., 2010).

Por otro lado, la capacidad antagonista de Trichoderma es muy variable, el micoparasitismo que presenta es un proceso complejo que incluye una serie de eventos sucesivos. Cuando el antagonista hace contacto físico con el fitopatógeno, sus hifas se enrollan alrededor de éste o se le adhieren por medio de estructuras especializadas (Manocha y Sahai, 1993; Cuervo-Parra et al., 2011a). La interacción que presenta Trichoderma con su huésped es específica y está controlada por lectinas, presentes en su pared celular. Las observaciones al microscopio electrónico muestran que Trichoder- ma produce y secreta enzimas micolíticas responsables de la degradación parcial de la pared celular de su huésped (Cuervo-Parra et al., 2011a).

Varios autores sugieren que el micoparasitismo es el mecanismo principal de acción de Trichoderma por medio del cual penetra a las células del patógeno causándole daño extensivo, como la degradación de la pared celular, la retracción de la membrana plasmática y la desorganización del citoplasma. También actúa sobre la replicación celular al inhibir la germinación de esporas y la elongación del tubo germinativo (Harman et al., 1981; Agamez et al., 2009).

Las especies de T. polysporum, T. viride y $T$. harzianum han mostrado un eficiente biocontrol contra diferentes aislados de Cladosporium herbarum (Barbosa et al., 2001). La confrontación directa entre T. harzianum contra Cladosporium sp. fue estudiada por Mokhtar y Dehimat (2012), quienes reportaron una inhibición del $57 \%$ al cuarto día, y por la acción de los compuestos volátiles producidos por T. harzianum a los 6 días. Por otra parte, Ahmad et al. (2012) reportaron la inhibición del crecimiento de Cladosporium spherospermun en un $72,2 \%$ en un periodo de 10 días.

En México la mayor parte de los trabajos se ha centrado en el estudio de los patógenos que causan enfermedades en el cultivo de cacao; es decir, Moniliophthora roreri y Phytophthora spp. (Phillips-Mora et al., 2006). En particular en la región Chontalpa, ubicada en el centro-oeste del estado de Tabasco, México, los hongos son los más agresivos con el cultivo. Desafortunadamente, existen pocos estudios enfocados en la identificación molecular de otras especies de hongos fitopatógenos que afectan las plantaciones. Por otro lado, desde el punto de vista biotecnológico, los sistemas de micoparasitismo son de gran importancia en los procesos ambientales. Sin embargo, la determinación de los parámetros cinéticos utilizando un modelo estructurado es un proceso complejo, debido a la interacción y medición de las concentraciones de metabolitos intermediarios, biomasa, ADN y enzimas involucradas (Villadsen et al., 2011). Por esta razón, los parámetros cinéticos más comúnmente utilizados se estiman a través de un modelo cinético no estructurado (López-Pérez et al., 2013).

Por lo tanto, existe la necesidad de un cono- 
cimiento satisfactorio que describa el comportamiento de la cinética del micoparasitismo basado en un modelo dinámico. Por lo cual, los objetivos de este estudio fueron (i) caracterizar molecularmente las cepas de hongos aislados de frutos de cacao, (ii) probar la actividad antagonista in vitro de Trichoderma harzianum VSL291 frente a los patógenos aislados de las plantas de cacao, y (iii) proponer un modelo cinético no estructurado basado en los datos experimentales del comportamiento del micoparasitismo.

\section{MATERIALES Y MÉTODOS}

\section{Aislamiento de las cepas de hongos}

Cada fragmento de fruto con síntomas de enfermedad se colocó en una cámara húmeda, posteriormente se incubó a $25^{\circ} \mathrm{C}$ durante 3 días en ausencia de luz. Las cepas fúngicas fueron aisladas de fragmentos de tejido enfermo, donde se observó crecimiento de micelio. Posteriormente, se tomó una parte del micelio crecido en cada uno de los tejidos enfermos con la ayuda de una aguja de disección y fueron resembrados en cajas Petri nuevas. Los cultivos puros se obtuvieron en medio PDA (Agar Papa Dextrosa) durante 5 días a temperatura ambiente. Las cepas se conservaron en una suspensión de esporas, en una solución de glicerol al $40 \%(\mathrm{v} / \mathrm{v})$ y se almacenaron a una temperatura de $-74^{\circ} \mathrm{C}$. Las cepas se identificaron como HT-ITV36, HT-ITV86, HT-ITV87, HT-ITV88 y HT-ITV89.

\section{Caracterización molecular}

La extracción de ADN de las cepas se realizó siguiendo la metodología propuesta por Kurzatkowski et al. (1996). Las secuencias de ADN, que contenían la región ITS I/5,8s/ITS II, se amplificaron por PCR utilizando los primers ITS1 e ITS4 (Foster et al., 1993; Kendall y Rygiewicz, 2005). Las amplificaciones por PCR se realizaron en un volumen total de reacción de $50 \mu \mathrm{L}$, que contenía: $10 \mu \mathrm{L}$ de buffer, $5 x$ Colorless, $200 \mu \mathrm{M}$ dNTPSs, $0,2 \mu \mathrm{M}$ de cada primer, 2,5 unidades de Taq ADN polimerasa, y 10-50 ng de ADN templado. Las reacciones de PCR se colocaron en un termociclador (TECHNE, modelo 3Prime, Bibby Scientific, Staffordshire, United Kingdom) bajo los siguientes parámetros: 5 min de desnaturalización inicial a $95^{\circ} \mathrm{C}$, seguido de 30 ciclos de 1 min de desnaturalización a $95^{\circ} \mathrm{C}, 1$ min de hibridación de los primers a $57^{\circ} \mathrm{C}, 1 \mathrm{~min}$ de extensión a $72^{\circ} \mathrm{C}$ y un periodo final de extensión de $12 \mathrm{~min}$ a $72^{\circ} \mathrm{C}$. Los productos resultantes se purificaron con el kit Geneclean ${ }^{\circledR}$ II (Bio101 Inc., California, EE.UU.) de acuerdo con el protocolo del fabricante. Las muestras de ADN fueron enviadas al Instituto de Biotecnología, Cuernavaca, Mor., México, para su secuenciación.

\section{Análisis de las secuencias}

Las secuencias de ADN obtenidas de las cepas amplificadas fueron ensambladas utilizando el programa Chromas 1,45 (School of Health Science, Griffith University, Gold Coast Campus, Southport, Queensland, Australia) y se alinearon con el programa ClustalX (Larkin et al., 2007). Las secuencias seleccionadas se sometieron a una búsqueda local con el programa BLASTN (Altschul et al., 1997) contra secuencias disponibles en el GenBank. El análisis filogenético de las secuencias alineadas se realizó por medio del análisis Neighbour-Joining y estuvo soportado por el método Bootstrap con 1000 repeticiones. El árbol filogenético sin raíz generado fue ensamblado con la ayuda del programa PhyloDraw 0,8 (PhyloDraw, 1999).

\section{Experimento de confrontación de Trichoderma vs C. cladosporioides}

Para el experimento de confrontación se utilizó como antagonista la cepa de T. harzianum VSL291 contra las cepas de C. cladosporioides, de acuerdo a la técnica propuesta por Szekeres et al. (2006). Discos de $5 \mathrm{~mm}$ de diámetro de micelio de $T$. harzianum y de $C$. cladosporioides incubados durante 3 días se sembraron en medio PDA en puntos equidistantes. Las cajas se incubaron a $25^{\circ} \mathrm{C}$ durante 7 días en ausencia de luz. Cuando se observó la actividad antagonista se tomaron las imágenes digitales a la misma distancia (18 $\mathrm{cm}$ ) con una cámara (Sony Cyber-shot DSC-P72). El porcentaje de inhibición se calculó utilizando el Indice de Biocontrol (IBC) de acuerdo con la fórmula descrita por Szekeres et al. (2006):

$$
\mathrm{IBC}=[\mathrm{A} / \mathrm{B}] \times 100,
$$

dónde $\mathrm{A}$ es el área de la colonia de T. harzianum y B es el área total ocupada por las colonias de $T$. harzianum y la cepa del hongo patógeno. El área del IBC se calculó con el software ImageJ (http:// rsbweb.nih.gov/).

\section{Micoparasitismo simulado T. harzianum - hongos fitopatógenos \\ Para determinar el micoparasitismo simulado} de T. harzianum sobre los hongos fitopatógenos se utilizó paredes celulares de C. cladosporioides, Colletotrichum gloeosporioides y Corynespora cassicola como única fuente de carbono. En matraces de $250 \mathrm{~mL}$ se colocó $50 \mathrm{~mL}$ de medio mínimo (conteniendo: $\mathrm{MgSO}_{4} \cdot 7 \mathrm{H}_{2} \mathrm{O}, 0,24 \mathrm{~g}$; $\mathrm{KCl}$, $0,24 \mathrm{~g} ; \mathrm{NH}_{4} \mathrm{NO}_{3}, 1,2 \mathrm{~g} ; \mathrm{ZnSO}_{4} \cdot 7 \mathrm{H}_{2} \mathrm{O}, 0,0024 \mathrm{~g}$; $\mathrm{MgCl}_{2} \cdot 7 \mathrm{H}_{2} \mathrm{O}, 0,0024 ; \mathrm{K}_{2} \mathrm{HPO}_{4}, 1,08 \mathrm{~g} ; \mathrm{FeSO}_{4} \cdot 7 \mathrm{H}_{2} \mathrm{O}$, $0,0024 \mathrm{~g}$, pH 5,5; para $1200 \mathrm{~mL}$ ) y $10 \mathrm{~g} \mathrm{~L}^{-1}$ de pared celular del micelio molido de C. cladosporioides, C. gloeosporioides y C. cassicola. Cada matraz 
se sembró con una azada de micelio de la cepa VSL291 de T. harzianum y se fermentó durante 3 días. Se tomó muestra cada $24 \mathrm{~h}$ y se determinó la actividad enzimática para $\beta$-1, 3-glucanasa (Nobe et al., 2003), xilanasa (Rawashdeh et al., 2005), quitinasa (Monreal y Reese, 1969), lipasa (Nawani et al., 1998) y proteasa (Kunitz, 1946). La cuantificación de azúcares reductores se realizó por el método descrito por Miller (1959). Cada experimento se realizó por triplicado. Para evaluar si existió diferencias entre cada uno de los tratamientos (actividades enzimáticas), el análisis estadístico de los datos se hizo mediante un diseño completamente aleatorio con tres repeticiones, usando un análisis de varianza (ANOVA) con el software Statistix9. La comparación de medias se realizó con la prueba de Tukey HSD All-Parwise a una diferencia significativa de $\mathrm{P} \leq 0,05$.

\section{RESULTADOS Y DISCUSIÓN}

\section{Aislamiento de cepas}

De un total de ciento veintiocho cepas de hongos aisladas de frutos de cacao con síntomas de moniliasis se identificaron cinco cepas como $C$. cladosporioides en base a sus características morfológicas (datos no mostrados). Los especímenes del género Cladosporium, ya han sido reportados por varios autores como patógenos de plantas como el cártamo (Carthamus tinctorius; Quintana-Obregón et al., 2011), tabaco (Nicotiana tabacum; Wang et al., 2014), remolacha azucarera (Beta vulgaris L. subsp. vulgaris var. altissima Döll; Robeson y Jalal, 1992), maíz (Zea mays; Orole y Adejumo, 2009), trigo (Triticum spp.; Jacyno et al., 1993) y cacao (Theobroma cacao; Amegbedzi, 2013). Esos hongos son los responsables de causar algunas enfermedades como las manchas foliares (Al-Sadi et al., 2014) y la enfermedad de la roña. Ésta última provoca la muerte de semillas y ramas, la pérdida de flores y disminuye la calidad del fruto (Goes, 1998) causando así pérdidas económicas en las plantaciones de cacao.

La enfermedad causada por estos hongos se diferencia de acuerdo a la planta que infectan y del tipo de síntomas. Por ejemplo, en los cereales, el hongo Cladosporium es responsable de causar la enfermedad llamada el punto negro de los cereales; en el género Cucurbita la roña de las cucurbitáceas; en la papaya (Carica papaya) la roña de la papaya; y en el tomate (Solanum lycopersicum) causa manchas marrones en las hojas (Kryczyński y Weber, 2011).

\section{Caracterización molecular}

Las cepas HT-ITV86 y HT-ITV88 mostraron una homología de 99\% con la cepa Cc_101 de C. cladosporioides (JX867558) en base a la región ITS
I/5,8s/ITS II. La secuencia de C. cladosporioides (KJ596320) aislada en la India, mostró una homología de 99\% con la cepa HT-ITV87. La secuencia de la cepa HT-ITV89 reveló su homología máxima (99\%) con la cepa NRRL 66016 de C. cladosporioides (KM030316), aislada en Estados Unidos de Norteamérica de plantas de maíz. Por último, la secuencia de la cepa HT-ITV36 reveló su homología máxima (99\%) con la cepa NRRL 52418 de C. cladosporioides (GQ221853), aislada de semillas de sorgo (Sorghum vulgare) en los Estados Unidos de Norteamérica. Los resultados obtenidos con el programa BLASTN confirman que las cepas HT-ITV36, HT-ITV86, HT-ITV87, HT-ITV88 y HT-ITV89 depositadas en las bases de datos del GenBank del National Center for Biotechnology Information (NCBI) con los números de acceso KF040480, KP963942, KP963943, KP963944 y KP9633945, corresponden a C. cladosporioides.

\section{Análisis filogenético}

Se realizó un análisis múltiple de secuencias de las cepas de $C$. cladosporioides obteniéndose la secuencia completa de la región ITS I/5,8s/ITS II, junto con los extremos de las subunidades de los genes $18 \mathrm{~S}$ y $28 \mathrm{~S}$. Las secuencias examinadas para la región ITS I/5,8s/ITS II, para las cepas de $C$. cladosporioides y otras secuencias de Cladosporium relacionadas del GenBank tuvieron una longitud de entre 830 a 1511 pb y la longitud de la región consenso para el alineamiento de todas las secuencias fue de $1518 \mathrm{pb}$.

Para el análisis de la subunidad 5.8S se observaron variaciones muy limitadas al cambio de unas pocas bases en las secuencias de C. cladosporioides aisladas del cacao, lo cual indicó que es una región muy conservada entre las secuencias de Cladosporium estudiadas. Por otro lado, en las subunidades $18 \mathrm{~S}$ y $28 \mathrm{~S}$, se observó una gran variabilidad, limitada a mutaciones puntuales, inserciones o delesiones, predominantemente entre las secuencias de los aislamientos de C. cladosporioides.

Dentro de las regiones ITS se observó variación en término de delesiones, inserciones y substituciones de bases, especialmente en la región ITS II, aunque muchas regiones conservadas estuvieron presentes en las regiones ITS I e ITS II. En general, la longitud de las secuencias para las regiones ITS I e ITS II, estuvo altamente conservada en todas las secuencias de las especies de Cladosporium, con variaciones limitadas al cambio de unas pocas bases.

El análisis filogenético de las secuencias de las cepas de C. cladosporioides aisladas del cacao y otras 15 secuencias de Cladosporium relacionadas procedentes del GenBank, generó un árbol por el método Neighbour Joining. El análisis filoge- 
nético de las 5 secuencias representativas de $C$. cladosporioides aisladas de frutos de cacao y otras secuencias de especies relacionadas de las bases de datos del GenBank formaron tres grupos mayores (I, II y III) soportados por valores estadísticos bootstrap altos de entre 700 a 1000 (Fig. 1).

En el grupo I se agruparon dos subgrupos (C1 y C2). El subgrupo C1 incluyó los aislados KP963942, KP963944 y JX867558, de los cuales las dos primeras corresponden a secuencias de $C$. cladosporioides aisladas de frutos de cacao, procedentes de México y la tercera de la India. El valor estadístico bootstrap alto que se obtuvo entre el alineamiento de la cepa HT-ITV88 (GenBank KP963944) y la secuencia de la cepa Cc_101 de C. cladosporioides (JX867558) confirmó la identificación de la cepa HT-ITV88. Por otro lado, aunque el valor estadístico bootstrap que se obtuvo para la cepa HT-ITV86 no fue muy alto (248) su alineamiento entre secuencias de $C$. cladosporioides dentro del subgrupo $\mathrm{C} 1$ muestra la probabilidad de pertenecer a esta especie.

Por su parte, en el subgrupo C2 se incluyó los aislados KP963943 y KJ596320, correspondientes a secuencias de C. cladosporioides de México y la
India, respectivamente. En este subgrupo la cepa HT-ITV87 se alineó junto a la secuencia de la cepa TSP1 de C. cladosporioides y se respaldó por un valor estadístico de soporte bootstrap de 523. Por otro lado, el grupo II se dividió en dos subgrupos (C3 y C4).

El subgrupo C3 incluyó los aislados KP963945, KM030316, FR837924 y GQ221852, todos de C. cladosporioides (2 Estados Unidos de Norteamérica, 1 México, 1 República Checa). En este subgrupo la cepa HT-ITV89 se alineó con la cepa NRRL 66016 de C. cladosporioides, con un valor de soporte de bootstrap de 1000. El subgrupo C4 incluyó los aislados EF679372, EF679390, EF679382 y EF679371, correspondientes a secuencias de C. macrocarpum, C. subtillissimum, C. ossifragi y C. macrocarpum (1 Turquía, 1 Estados Unidos de Norteamérica, 1 Noruega y 1 Rumania), cuyos alineamientos estuvieron soportados por valores estadísticos bootstrap altos. Por último, el grupo III se dividió en tres subgrupos (C5, C6 y C7).

El subgrupo C5 incluyó los aislados KF040480, GQ221853 y HQ130717, con dos secuencias de C. cladosporioides y una secuencia de Cladosporium sp. procedentes de México, Estados Unidos de

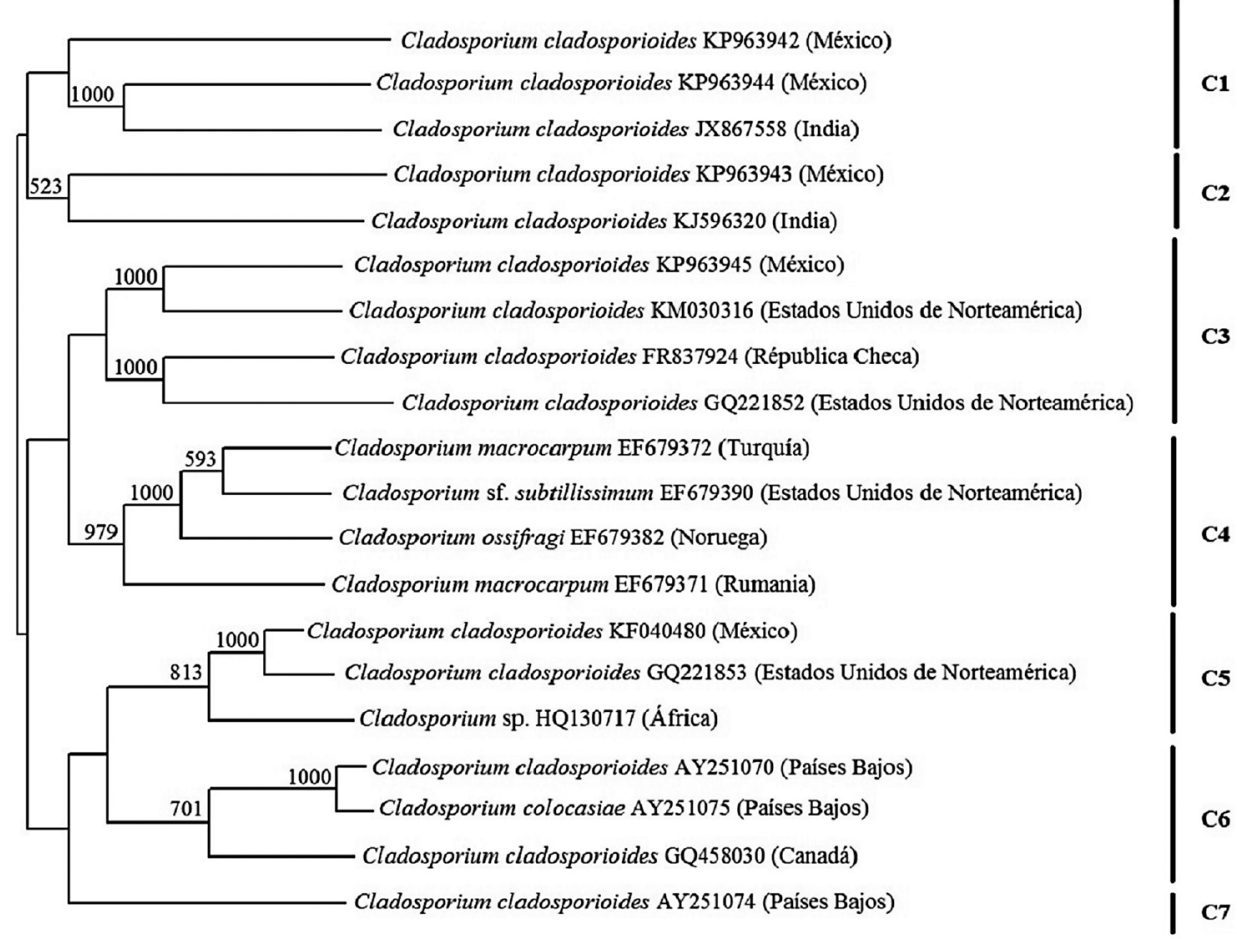

Fig. 1. Árbol filogenético generado por el método Neighbour Joining derivado de los datos de las secuencias de los hongos para la región ITS I/5,8s/ITS II del ARN ribosomal. Los números adyacentes a los puntos de ramificación son los valores bootstrap para 1000 repeticiones.

Fig. 1. Phylogenetic tree generated by the Neighbour Joining method derived from the sequence data for fungal ITS I/5.8s/ITS II region of the RNAr. Numbers adjacent to branch points are bootstrap values from 1000 interactions. 
América y África, respectivamente. En este subgrupo la secuencia de la cepa HT-ITV36 se alineó con la secuencia de la cepa NRRL 52418 de C. cladosporioides, soportado por un valor estadístico bootstrap de 1000. Dicho resultado estadístico confirma que ésta cepa pertenece a la especie $C$. cladosporioides. El subgrupo C6 incluyó los aislados AY251070, AY251075 y GQ458030 de los cuales la mayoría son secuencias de cepas de $C$. cladosporioides procedentes de Países Bajos y Canadá, soportados por valores estadísticos altos. Por último, el subgrupo C7 incluyó solo el aislado AY251074 procedente de Países Bajos.

\section{Experimento de confrontación}

El experimento de confrontación mostró una zona de inhibición progresiva producida por $T$. harzianum VSL291 en contra de C. cladosporioides (datos no mostrados), que se incrementó a través del tiempo, acompañada por el ataque del micelio del hongo patógeno (Tabla 1). T. harzianum mostró un efecto marcado de híperparasitismo, que fue observado por el crecimiento sobre la colonia del fitopatógeno y por la competencia por espacio y nutrientes (Ezziyyani et al., 2004).

La aplicación de cepas de T. harzianum en frutas de uvas y manzanas ha sido reportada reduciendo parcialmente la enfermedad causada por el hongo Botrytis cinerea y las protegió por más de dos meses, e igualmente mostró efecto sobre Penicillium expansum (Latorre et al., 1997). Así mismo, se observó que T. harzianum y T. pseudokoningi controlaron el desarrollo de Monilinia laxa en ciruela de hueso y B. cinerea en manzanas (Velázquez-del Valle et al., 2008).

\section{Simulación de micopararasitismo}

T. harzianum secretó en el medio de cultivo algunas enzimas como la $\beta$-1,3-glucanasas $(0.58$
$\left.\mathrm{mU} \mathrm{mg} \mathrm{m}^{-1}\right)$, quitinasas $\left(0.08 \mathrm{mU} \mathrm{mg}^{-1}\right)$, xilanasas $\left(0.31 \mathrm{mU} \mathrm{mg}^{-1}\right)$, proteasas $\left(0.21 \mathrm{mU} \mathrm{mg}^{-1}\right)$ y lipasas $\left(0.15 \mathrm{mU} \mathrm{mg}^{-1}\right)$, en presencia de paredes celulares de las cepas HT-ITV36, HT-ITV86, HT-ITV87, HT-ITV88 y HT-ITV89 de C. cladosporioides, y de las cepas HT-ITV41 y HT-ITV32 de C. gloeosporioides y $C$. cassicola, respectivamente. La actividad micoparasítica de Trichoderma dependió de la secreción de una mezcla compleja de enzimas hidrolíticas capaces de degradar las paredes celulares de los hongos patógenos (Suarez et al., 2005). En muchos miembros del género Trichoderma se ha demostrado su acción antagónica contra patógenos de plantas y la producción de enzimas líticas (Haggag et al., 2006).

Para la producción de quitinasas se observó que a las 12 y $24 \mathrm{~h}$ se produjo la actividad enzimática mayor $\left(0,078 \mathrm{mU} \mathrm{mg}^{-1} \pm 0,01\right.$ y $0,054 \mathrm{mU}$ $\mathrm{mg}^{-1} \pm 0,02$ de proteína, respectivamente) y para C. gloeosporioides y C. cassicola $\left(0,084 \mathrm{mU} \mathrm{mg}^{-1} \pm\right.$ 0,01 y $\left.0,024 \mathrm{mU} \mathrm{mg}^{-1} \pm 0,015\right)$. De la Cruz et al. (1992) obtuvieron valores de actividad específica de entre 670 y $800 \mathrm{mU} \mathrm{mg}^{-1}$ de proteína para la actividad quitinasa con la cepa CECT 2413 de T. harzianum, crecida con paredes celulares de B. cinerea, Gibberella fujikuroi, Rhizoctonia solani, Phytophthtora citrophthora y T. harzianum, que son más altos a los valores obtenidos en este trabajo. Kucuk y Kivanc (2008) produjeron quitinasas en medios de cultivo líquidos que contenían paredes celulares de Gibberella zeae y Aspergillus ustus como única fuente de carbono con cepas de T. harzianum y reportaron valores similares a los encontrados en este trabajo.

La producción máxima de xilanasas por $T$. harzianum VSL291 se observó a las 12 h $(0,3 \mathrm{mU}$ $\mathrm{mg}^{-1}$ de proteína $\left.\pm 0,003\right)$ y se mantuvo constante a una concentración de 0,25 hasta las 96 horas y para C. gloeosporioides y C. cassicola $(0,083 \mathrm{mU}$

Tabla 1. Índice de Biocontrol (IBC) de la cepa VSL 291 contra C. cladosporioides Table 1. Biocontrol index (IBC) of VSL 291 strain against $C$. cladosporioides

\begin{tabular}{cccc}
\hline Tiempo de incubación (h) & Área $^{\mathrm{a}} \mathbf{T} \mathbf{~ c m}^{-\mathbf{2}} \pm \mathbf{D E}$ & Área $^{\mathbf{b}} \mathbf{T}+\mathbf{P} \mathbf{~ c m}^{-2} \pm \mathbf{D E}$ & IBC $\mathbf{~} \mathbf{\%})$ \\
\hline 0 & 0 & 0 & 0 \\
24 & $10,98 \pm 0,58$ & $20,78 \pm 0,50$ & 52,83 \\
48 & $16,34 \pm 1,13$ & $27,84 \pm 1,18$ & 58,69 \\
72 & $18,23 \pm 2,21$ & $29,23 \pm 2,20$ & 62,37 \\
96 & $19,68 \pm 1,29$ & $31,18 \pm 0,73$ & 63,12 \\
120 & $23,34 \pm 0,63$ & $35,29 \pm 1,09$ & 66,14 \\
144 & $30,12 \pm 0,78$ & $42,31 \pm 0,33$ & 71,18 \\
168 & $43,03 \pm 1,21$ & $56,22 \pm 0,07$ & 76,29 \\
\hline
\end{tabular}

\footnotetext{
a Área T: área total de la colonia de Trichoderma.

${ }^{\mathrm{b}}$ Área T + P: área total ocupada por las colonias de Trichoderma y el hongo patógeno.

${ }^{\mathrm{C}}$ IBC del crecimiento del hongo patógeno.
} 
$\mathrm{mg}^{-1} \pm 0,01$ y $\left.0,133 \mathrm{mU} \mathrm{mg}^{-1} \pm 0,05\right)$. Stricker et al. (2006), encontraron que la cepa QM9414 de Hypocrea jecorina produjo la actividad máxima de xilanasa $\left(0,206 \pm 0,015 \mathrm{U} \mathrm{mg}^{-1}\right.$ de proteína) a las 48 h utilizando xilano como fuente de carbono. Sin embargo, Seiboth et al. (2003) evaluaron la producción de xilanasa con $H$. jecorina utilizando seis sustratos diferentes, encontrando que la mejor actividad específica $\left(0,33 \pm 0,05 \mathrm{U} \mathrm{mg}^{-1}\right)$ se obtuvo cuando $H$. jecorina creció en el medio conteniendo xilitol como sustrato. Ujiie et al. (1991) reportaron una actividad específica de xilanasa de 24,4 U $\mathrm{mg}^{-1}$ de proteína para la enzima cruda producida por Trichoderma viride. Otros autores evaluaron las actividades específicas de xilanasa producidas por cepas nativas y recombinantes (ATX2-12, ATX2-16 y TU-6) de Trichoderma reesei en medio suplementado con glucosa y xilano, y reportan que las actividades específicas de xilanasa a partir de xilano por las cepas ATX2-12, ATX2- 16 y TU-6 fueron 30, 27 y $24 \mathrm{U} \mathrm{mg}^{-1}$ de proteína, respectivamente (Kurzatkowski et al., 1996). Rezende et al. (2002) reportaron una actividad específica de xilanasa de $13 \mathrm{U} \mathrm{mg}^{-1}$ de proteína. Los resultados obtenidos en los trabajos anteriores son superiores a los mostrados en este estudio, ya que en esos trabajos se utilizaron fuentes de carbono específicas para la producción de xilanasas.

Para el caso de las proteasas, la actividad máxima se detectó a las 24 h (0,220 mU Tirosina $\mathrm{mg}^{-1}$ proteína $\pm 0,003$ ) observándose una pérdida de $0,150 \mathrm{mU} \pm 0,009$ a las $48 \mathrm{~h}$ y para $C$. gloeosporioides y $C$. cassicola $\left(0,068 \mathrm{mU} \mathrm{mg}^{-1} \pm 0,01\right.$ y 0,06 $\mathrm{mU} \mathrm{mg}^{-1} \pm 0,015$, respectivamente). La secreción proteolítica de T. harzianum T39 mostró efecto directo sobre $B$. cinérea reduciendo la germinación y el desarrollo de la enfermedad (Elad y Kapat, 1999). La actividad máxima para lipasas se detectó entre las $12 \mathrm{~h}$ y $24 \mathrm{~h}$ de incubación $(0,154 \mathrm{mU}$ $p$-nitrofenol $\mathrm{mg}^{-1}$ proteína \pm 0,08 y 0,09 mU p-nitrofenol $\mathrm{mg}^{-1}$ proteína $\pm 0,09$, respectivamente) y para C. gloeosporioides y C. cassicola $(0,0754 \mathrm{mU}$ $\mathrm{mg}^{-1} \pm 0,01$ y $\left.0,094 \mathrm{mU} \mathrm{mg}^{-1} \pm 0,015\right)$. Chet (1987) encontró lipasas en el medio de cultivo donde creció $T$. harzianum en presencia de micelio de $S$. rolfsii. Kashmiri et al. (2006) encontraron que la actividad máxima específica de lipasa producida por $T$. viride durante la fermentación fue de $328 \mathrm{U}$ $\mathrm{mg}^{-1}$ de proteína a las $48 \mathrm{~h}$, similar a la actividad de lipasa máxima observada en éste estudio utilizando las paredes celulares de C. cladosporioides pero con valores más bajos de actividad enzimática. Las enzimas producidas por T. harzianum degradaron las paredes celulares de C. cladosporioides a partir de las $50 \mathrm{~h}$ (Fig. 2).

\section{Modelado en bioprocesos}

Los modelos no estructurados describen algunos componentes, tales como sustratos principales, microorganismos, productos de interés, que

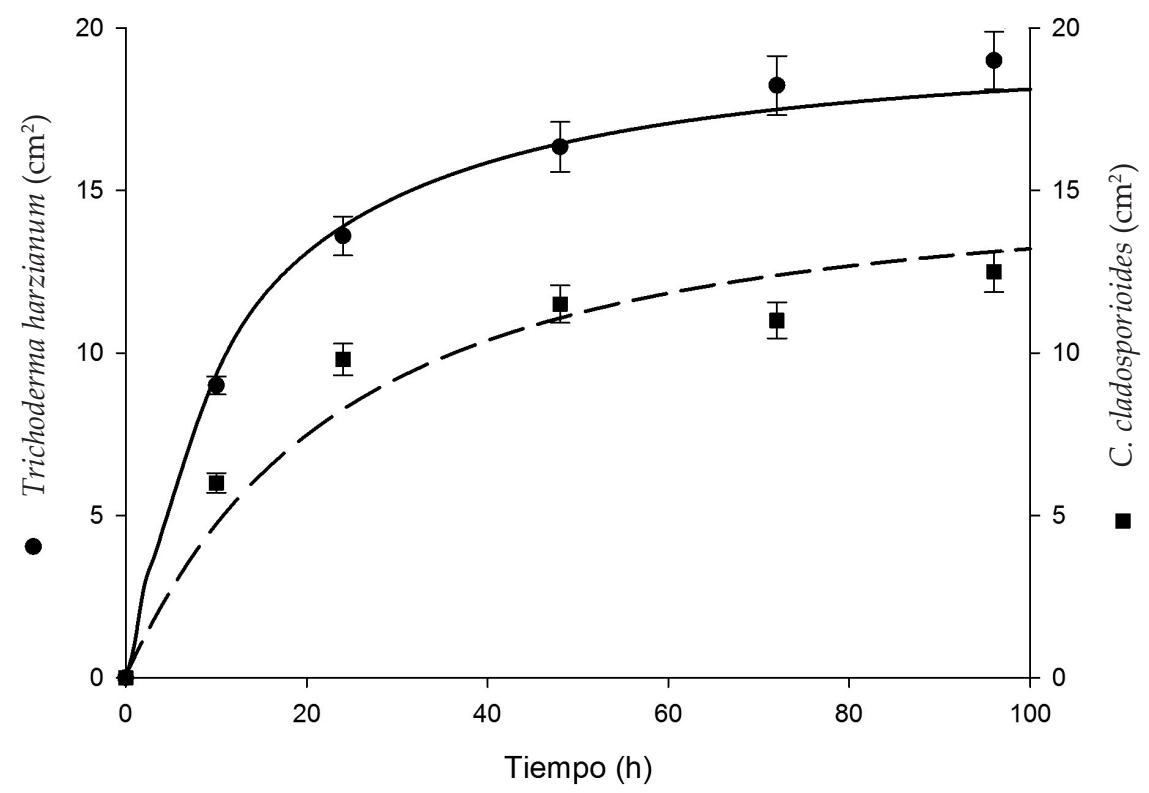

Fig. 2. Comportamiento dinámico de los datos experimentales (símbolos) y la predicción del modelo (línea) para T. harzianum y C. cladosporioides.

Fig. 2. Dynamic behavior of the experimental data (symbols) and the prediction model (line) for $T$. harzianum and C. cladosporioides. 
permiten limitar el número de variables y parámetros del sistema (Dochain, 2003). En la fermentación por lotes, la dinámica del sistema puede ser descrito por el siguiente modelo:

$$
\frac{d x(t)}{d t}=K \tau(x(t))
$$

dónde

$x \in \mathfrak{R}_{+}^{n}$ es el vector de concentraciones; $K \in \mathfrak{R}_{+}^{n x m}$ es la matriz de coeficientes estequiométricos ( $\mathrm{m}$ $<\mathrm{n}) ; \tau_{i} \in \mathfrak{R}_{+}^{n}$ es el vector de términos cinéticos; $t \in \mathfrak{R}_{+}$es el tiempo; y

$$
\tau(x(t))=\mu(\mathrm{t}) \mathrm{x}(\mathrm{t})
$$

dónde $\mu(t)$ es la tasa de crecimiento específico que es una función de diferentes concentraciones de acuerdo al sistema en estudio.

Tales velocidades de reacción varían con el tiempo y por lo general se ven influidas por muchos factores físico-químicos y biológicos ambientales como el sustrato, los microorganismos y las concentraciones de productos, así como el $\mathrm{pH}$, la temperatura, la concentración de oxígeno disuelto o varios inhibidores del crecimiento microbiano. Los cuales, para este trabajo representan las concentraciones de las actividades enzimáticas de lipasas, glucanasas, proteasas, quitinasas, y xilanasas, de T. harzianum con paredes celulares de C. cladosporioides.

\section{Modelo cinético propuesto}

El modelo cinético propuesto representa el mecanismo de control biológico mediante la velocidad de crecimiento de los hongos y la secreción de enzimas. El modelo se basó en el balance de materia vía cinéticas no estructuradas represen- tadas por ecuaciones diferenciales en función del tiempo (modelo dinámico). Para la solución del modelo se diseñó una red de programación gráfica (Model Maker ${ }^{\circledR)}$ representando el mecanismo de reacción del micoparasitismo en función de las enzimas involucradas. Para la solución numérica se utilizó el método de Runge-Kutta. Con la resolución de las ecuaciones (4) a la (10), se obtuvieron 34 parámetros que permitieron caracterizar el comportamiento del micoparasitismo simulado. Los parámetros se ajustaron a los datos experimentales y se validaron mediante el cálculo de un índice de desempeño global (ecuación 3) lo cual indicó un ajuste satisfactorio (Fig. 3 y Fig. 4).

$$
\Delta=1-\frac{\left.\sum_{\mathrm{i}=1}^{\mathrm{N}}\left[\Delta\left(\mathrm{t}_{\mathrm{i}}\right)-\mathrm{M}\left(\mathrm{t}_{\mathrm{i}}\right)\right]\right]}{\sum_{\mathrm{i}=1}^{\mathrm{N}}\left|\mathrm{M}\left(\mathrm{t}_{\mathrm{i}}\right)-\mathrm{M}\right|}
$$

dónde $\Delta\left(t_{i}\right)$ es el valor numérico simulado variable a tiempo $t_{i} ; M\left(t_{i}\right)$ el valor observado de la misma variable en el tiempo $t_{i} ; \mathrm{M}^{*}$ es el valor medio de la variable observada, $\mathrm{y} \mathrm{N}$ es el número de datos. $\Delta$ varía entre $(-\infty, 1)$ en dónde un valor positivo de $\Delta$ representa un simulación aceptable mientras que $\Delta>0,5$ representa un buena simulación.

\section{Modelo dinámico}

Balance de T. harzianum (S):

$$
\frac{d S}{d t}=\frac{\mu_{\max }}{Y} *\left(1-\frac{S}{k_{s}}\right)^{\alpha} *\left[\frac{X}{k_{x}+X}\right]
$$

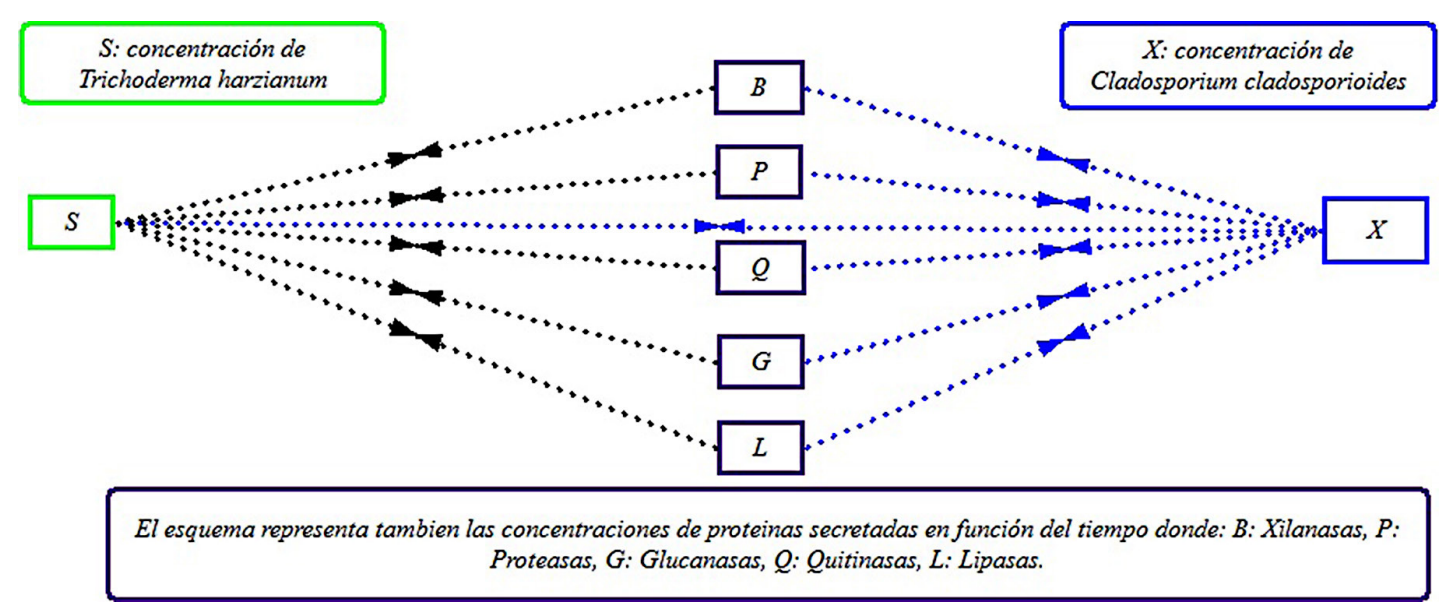

Fig. 3. Red de programación gráfica para el micoparasitismo.

Fig. 3. Graphical network programming for mycoparasitism. 


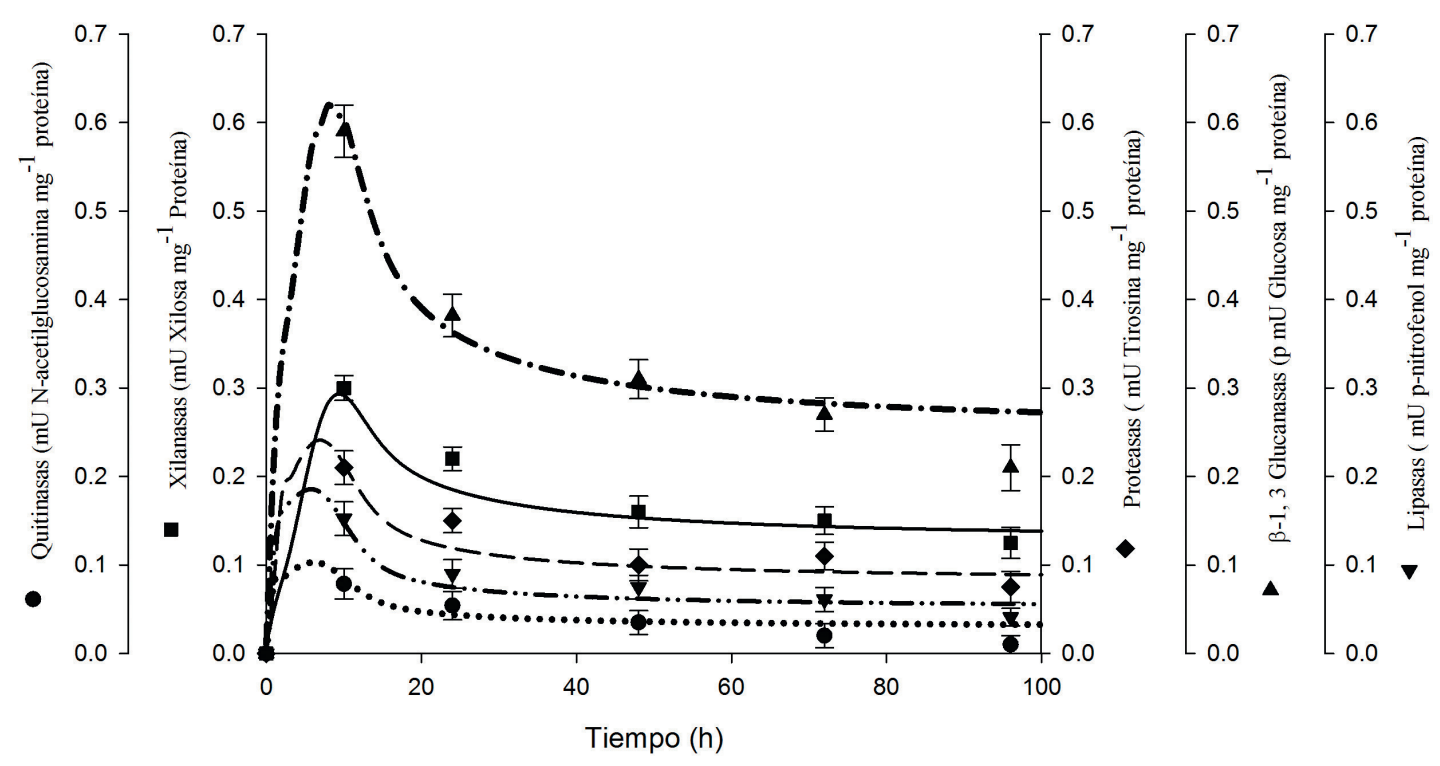

Fig. 4. Comportamiento dinámico de los datos experimentales (símbolos) y la predicción del modelo (línea) para xilanasas, proteasas, $\beta-1,3$ glucanasas, quitinasas y lipasas.

Fig. 4. Dynamic behavior of the experimental data (symbols) and the prediction model (line) for xylanases, proteases, $\beta-1,3$ glucanases, chitinases and lipases.

Balance de C. cladosporioides (X):

$$
\frac{d X}{d t}=\mu_{\max } *\left(1-\frac{S}{k_{s}}\right)^{\alpha} *\left[\frac{X}{k_{x}+X}\right] *\left[\frac{\varphi_{1}}{\varphi_{1}+Q}\right] *\left[\frac{\varphi_{2}}{\varphi_{2}+P}\right] *\left[\frac{L}{\varphi_{3}+L}\right] *\left(\frac{B}{G+\varphi_{4}}\right)
$$

dónde:

$\mu_{\max }=0,0156$ Velocidad máxima de crecimiento $\left[\mathrm{cm}^{2} \mathrm{~h}^{-1}\right] ; k_{s}=0,25$ término de inhibición para el modelo Luong $\left[\mathrm{cm}^{2}\right] ; k_{x}=15$ Constante de afinidad de T. harzianum $\left[\mathrm{cm}^{2}\right] ; \alpha=1,6$ Término exponencial para el modelo Luong [adimensional]; $Y=0,8$ Rendimiento [adimensional]; $\varphi=2,3$ constante de modelo de Bulton para quitinasas sobre $C$. cladosporioides [ $\left.\mathrm{mU} \mathrm{mg}^{-1}\right] ; \varphi_{2}=3,9$ constante de modelo de Bulton para proteasas sobre $C$. cladosporioides $\left[\mathrm{mU} \mathrm{mg}^{-1}\right] ; \varphi_{3}=0,01$ constante de modelo de Bulton para lipasas sobre $C$. cladosporioides $\left[\mathrm{mU} \mathrm{mg}^{-1}\right.$; $\varphi_{4}=0,01$ constante de modelo de Bulton para $\beta-1,3$ glucanasas sobre $C$. cladosporioides [ $\mathrm{mU} \mathrm{mg}^{-1}$ ]

Balance de xilanasas $(B)$ :

$$
\frac{d B}{d t}=\left[\lambda_{B} *\left(1-\frac{B}{k_{B}}\right)^{\beta}-k_{d} * X * B+\lambda_{1} * \exp ^{\left(-\lambda_{2} * X^{*} t\right)}-\lambda_{1} * \exp \left(-\lambda_{3} * X^{*} t\right)\right] * S
$$


dónde:

$\lambda_{B}=0,015$ velocidad especifica de producto de xilanasas $\left[\mathrm{mU} \mathrm{mg}^{-1} \mathrm{~h}^{-1} \mathrm{~cm}^{-2}\right] ; k_{\mathrm{s}}=13$ término de inhibición para el modelo Luong para xilanasas $\left[\mathrm{mU} \mathrm{mg}^{-1}\right] ; \beta=3,12$ Término exponencial para el modelo Luong [adimensional]; $k_{d}=0,01$ constante de decaimiento referida a C. cladosporioides $\left[\mathrm{h}^{-1}\right.$ $\left.\mathrm{cm}^{-2}\right]$. También fue reportada por Cunniffe y Gilligan (2011) con un valor de 1 a $5, \lambda_{1}=3,3$ constante referida al metabolismo de xilanasas $\left[\mathrm{mU} \mathrm{mg}^{-1} \mathrm{~h}^{-1}\right.$ $\left.\mathrm{cm}^{-2}\right] ; \lambda_{2}=1,36$ constante exponencial de la actividad de xilanasa (activas) [adimensional]; $\lambda_{3}=2,0$ constante exponencial de la actividad de xilanasa (inactivas) [adimensional],

Balance de proteasas $(P)$ :

$$
\frac{d P}{d t}=\left[\rho_{P} *\left(1-\frac{P}{k_{P}}\right)^{\chi}-k_{d} * X * P+\rho_{1} * \exp ^{\left(-\rho_{2} * X * t\right)}\right] * S
$$

dónde:

$\rho_{p}=0,024$ velocidad especifica de producto de proteasas [mU mg-1 $\mathrm{h}^{-1} \mathrm{~cm}^{-2}$; $k_{p}=16$ término de inhibición para el modelo Luong para proteasas $\left[\mathrm{mU} \mathrm{mg}^{-1}\right] ; X=1,6$ Término exponencial para el modelo Luong [adimensional]; $\rho_{1}=0,2$ constante referida al metabolismo de proteasas $\left[\mathrm{mU} \mathrm{mg}^{-1} \mathrm{~h}^{-1}\right.$ $\left.\mathrm{cm}^{-2}\right] ; \rho_{2}=1,5$ constante exponencial de la actividad de proteasas [adimensional].

Balance de quitinasas $(Q)$ :

$$
\frac{d Q}{d t}=\left[\kappa_{Q} *\left(1-\frac{Q}{k_{Q}}\right)^{\delta}-k_{d} * X * Q+\kappa_{1} * \exp ^{\left(-\kappa_{2} * X^{*} t\right)}\right] * S
$$

dónde:

$K_{Q}=0,0056$ velocidad especifica de producto de quitinasas $\left[\mathrm{mU} \mathrm{mg}^{-1} \mathrm{~h}^{-1} \mathrm{~cm}^{-2}\right] ; K_{Q}=15$ término de inhibición para el modelo Luong para quitinasas $\left[\mathrm{mU} \mathrm{mg}^{-1}\right] ; \delta=1,6$ Término exponencial para el modelo Luong [adimensional]; $\kappa_{1}=1,2$ constante referida al metabolismo de quitinasas $\left[\mathrm{mU} \mathrm{mg}^{-1}\right.$ $\left.\mathrm{h}^{-1} \mathrm{~cm}^{-2}\right] ; \kappa_{2}=3,5$ constante exponencial de la actividad de quitinasas [adimensional].

Balance $\beta-1,3$ glucanasas $(G)$ :

$$
\frac{d G}{d t}=\left[v_{G} *\left(1-\frac{G}{k_{G}}\right)^{\varepsilon}-k_{d} * X * G+v_{1} * \exp ^{\left(-v_{2} * X^{*} t\right)}\right] * S
$$


dónde:

$V_{G}=0,048$ velocidad especifica de producto de glucanasas $\left[\mathrm{mU} \mathrm{mg}^{-1} \mathrm{~h}^{-1} \mathrm{~cm}^{-2}\right] ; k_{G}=15$ término de inhibición para el modelo Luong para glucanasas $\left[\mathrm{mU} \mathrm{mg}^{-1}\right] ; \varepsilon=1,6$ Término exponencial para el modelo Luong [adimensional]; $V_{1}=1,25$ constante referida la metabolismo de glucanasas $\left[\mathrm{mU} \mathrm{mg}^{-1}\right.$ $\left.\mathrm{h}^{-1} \mathrm{~cm}^{-2}\right] ; V_{2}=1,4$ constante exponencial de la actividad de glucanasas [adimensional].

Balance de lipasas $(L)$ :

$$
\frac{d L}{d t}=\left[\sigma_{L} *\left(1-\frac{L}{k_{L}}\right)^{\eta}-k_{d} * X * L+\sigma_{1} * \exp ^{\left(-\sigma_{2} * X * t\right)}\right] * S
$$

dónde:

$\sigma_{L}=0,001$ velocidad especifica de producto de lipasas $\left[\mathrm{mU} \mathrm{mg}^{-1} \mathrm{~h}^{-1} \mathrm{~cm}^{-2}\right] ; k_{L}=15$ término de inhibición para el modelo Luong para lipasas $\left[\mathrm{mU} \mathrm{mg}^{-1}\right] ; \quad \varepsilon=1,6$ Término exponencial para el modelo Luong [adimensional]; $\sigma_{1}=0,63$ constante referida al metabolismo de lipasas $\left[\mathrm{mU} \mathrm{mg}^{-1} \mathrm{~h}^{-1}\right.$ $\left.\mathrm{cm}^{-2}\right] ; \sigma_{2}=1,4$ constante exponencial de la actividad de lipasas [adimensional].

Cabe mencionar que muchas de estas constantes están en rango con algunos experimentos relacionados con el micoparasitismo (Beg et al., 2001; Cai et al., 2004; Lu et al., 2008; Knob y Carmona, 2010). En la literatura solo se tienen reportes de cinéticas referidas a los hongos antagonistas y/o patógenos sin incluir el efecto de las proteínas en el modelado cinético (Beg et al., 2001; Cunniffe y Gilligan, 2011).

\section{CONCLUSIONES}

Las técnicas de caracterización molecular permitieron identificar a las secuencias de las cepas HT-ITV36, HT-ITV86, HT-ITV87, HT-ITV88 y HT-ITV89 como especies de C. cladosporioides sensu lato. Por otro lado, la actividad enzimática de T. harzianum VSL291 contra C. cladosporioides, C. gloeosporioides y C. cassicola se relacionó con el aumento de las actividades enzimáticas. Además, los valores del IBC obtenidos para la cepa de T. harzianum VSL291, sugieren que las cepas de Trichoderma aisladas de $T$. cacao tiene la capacidad para antagonizar de manera más eficiente a los patógenos fúngicos de este cultivo. Finalmente, el modelo cinético propuesto predijo las concentraciones de las enzimas $\beta-1,3$ glucanasas, quitinasas, lipasas, proteasas, xilanasas producidas por Trichoderma en paredes celulares de C. cladosporioides, C. gloeosporioides y C. cassicola. Además, este modelo puede utilizarse como una herramienta para la estimación de actividades enzimáticas con un enfoque de instrumentación virtual.

\section{LITERATURA CITADA}

Agamez, R.E., V.J. Barrera, y L.Z. Oviedo. 2009. Evaluación del antagonismo y multiplicación de Trichoderma sp. en sustrato de plátano en medio líquido estático. Acta Biol. Colomb. 14:61-70.

Al-Sadi, A.M., Z.A. Al-Alawi, M.L. Deadman, and P. Annette. 2014. Etiology of four foliar and root diseases of wild plants in Oman. Can. J. Plant Pathol. 36:514-522.

Altschul, S.F., T.L. Madden, A.A. Schaffe, J. Zhang, Z. Zhang, W. Milleer, and D.J. Lipman. 1997. Grapped BLAST and PSI-BLAST: a new generation of protein database search programs. Nucl. Acids Res. 25(17):3389-3402.

Amegbedzi, M.A. 2013. Effects of two fungicides, Caocobre and Ridomil on the rhizosphere microflora of cocoa (Theobroma cacao L) seedlings. Int. J. Pure Appl. Sci. Technol. 15(1):3142.

Ahmad, M.L., W.M. Rafiq, S.A. Sheikh, S. Sahay, and D.M. Suliman. 2012. Antagonistic potenciality of Trichoderma harzianum against Cladosporium spherospermum, Aspergillus niger and Fusarium oxysporum. J. Biol. Agric. and Healthc. 2(8):72-76.

Barbosa, M.A.G., K.G. Rehn, M. Menezes, and R.L. Mariano. 2001. Antagonism of Trichoderma species on Cladosporium herbarum and their enzimatic characterization. Braz. J. Microbiol. 32:98-104.

Beg, Q.K., M. Kapoor, L. Mahajan, and G.S. Hoondal. 2001. Microbial xylanases and their industrial applications: A review. Appl. Microbiol. Biotechnol. 56:326-338. 
Bensch, K., J.Z. Groenewald, J. Dijksterhuis, M. Starink-Willemse, B. Andersen, B.A. Summerell, et al. 2010. Species and ecological diversity within the Cladosporium cladosporioides complex (Davidiellaceae, Capnodiales). Stud. Mycol. 67:1-94.

Braun, U., P.W. Crous, and K. Schubert. 2008. Taxonomic revision of the genus Cladosporium s. lat. 8. Reintroduction of Graphiopsis (= Dichocladosporium) with further reassessments of Cladosporioid hyphomycetes. Mycotaxon. 103:207-216.

Cai, Q., X. Yue, and T. Niu. 2004. The screening of culture condition and properties of xilanasas by white-rot fungus Pleurotus ostreatus. Process Biochem. 39:1561-1566.

Chet, I. 1987. Trichoderma - Aplication, mode of action and potential as a biocontrol agent of soil borne plant pathogenic fungi. p. 137160. In I. Chet (ed.) Innovative Approaches to Plant Disease Control. Wiley, New York, USA.

Cuervo-Parra, J.A., M. Ramírez-Suero, V. Sánchez-López, and M. Ramírez-Lepe. 2011a. Antagonistic effect of Trichoderma harzianum VSL 291 on phytopathogenic fungi isolated from cocoa (Theobroma cacao L.) fruits. Afr. J. Biotechnol. 10(52):10657-10663.

Cuervo-Parra, J.A. V. Sánchez-López, M. Ramirez-Suero, and M. Ramírez-Lepe. 2011b. Morphological and molecular characterization of Moniliophthora roreri causal agent of frosty pod rot of cocoa tree in Tabasco, Mexico. Plant Pathol. 10(3):122-127.

Cunniffe, N.J., and C.A. Gilligan. 2011. A theoretical framework for biological control of soilborne plant pathogens: Identifying effective strategies. J. Theor. Biol. 278:32-43.

De la Cruz J., A. Hidalgo-Gallego, J.M. Lora, T. Benitez, J.A. Pintor-Toro, and A. Llobell. 1992. Isolation and characterization of three chitinases from Trichoderma harzianum. Eur. J. Biochem. 206:859-867.

Djocgoue, P.F., T. Boudjeko, D.J. Nankeu, M.I.B. Efombagn, S. Nyasse, and D.N. Omokolo. 2006. Comparative assessment of the resistance of cocoa (Theobroma cacao L.) progenies from SNK10 x SNK413; ICS84 x ICS95 to Phytophthora megakarya in Cameroon by measuring size of necrotic lesion along the midrib. Plant Pathol. 5:329-335.

Dochain, D. 2003. State and parameter estimation in chemical and biochemical processes: a tutorial. J. Process Contr.13:801-818.

Elad, Y., and A. Kappat. 1999. Role of Trichoderma harzianum protease in the biocontrol of Botrystis cinerea. Eur. J. Plant Pathol. 105:177189.
Ezziyyani, M., S.C. Pérez, A.A. Sid, M.E. Requena, y M.E. Candela. 2004. Trichoderma harzianum como biofungicida para el biocontrol de Phytophthora capsici en plantas de pimiento (Capsicum annuum L.). Anales de Biología 26:35-45.

Foster, D.M., K.R. Kozak, M.G. Loftus, J.J. Stevens, and I.K. Ross. 1993. The polymerase chain reaction and its application to filamentous fungi. Mycol. Res. 97:769-781.

Goes, A. 1998. Donças fúngicas. p. 208-216. In Simposio Brasileiro sobre a Cultura do Maracujazeiro. 10-13 fevreiro 1998. Faculdade de Ciencias Agrárias e Veternárias, Universidade Estadual Paulista, Jaboticabal, Brasil.

Haggag, M.W., L.A. Kansohy, and M.A. Aly. 2006. Characterization and antifungal activity against brown spot disease on faba bean. Plant Pathology Bulletin 15:231-239.

Harman, G., I. Chet, and R. Baker. 1981. Factors affecting Trichoderma hamatum applied to seed as a biocontrol agent. Phytopathol. 71:569-572.

Jacyno, J.M., J.S. Harwood, and M.K. Lee. 1993. Isocladosporin, a biologically active isomer of cladosporin from Cladosporium cladosporioides. J. Nat. Prod. 56(8):1397-1401.

Kashmiri, M.A., A. Adnan, and B.W. Butt. 2006. Production, purification and partial characterization of lipase from Trichoderma viride. Afr. J. Biotechnol. (10):878-882.

Kendall, J.M., and P.T. Rygiewicz. 2005. Fungal-specific PCR primers developed for analysis of the ITS region of environmental DNA extracts. BMC Microbiol. 5:1-11.

Kilaru1, A., B.A. Bailey, and K.H. Hasenstein. 2007. Moniliophthora perniciosa produces hormones and alters endogenous auxin and salicylic acid in infected cocoa leaves. FEMS Microbiol. 274:238-244.

Knob, A., and E.C. Carmona. 2010. Purification and characterization of two extracellular $\mathrm{xy}$ lanases from Penicillium sclerotiorum: A novel acidophilic xylanase. Appl. Biochem. Biotechnol. 162:429-443.

Kryczyński, S., and Z. Weber. 2011. Fitopatologia. Tome 2. Choroby roślin uprawnych. Powszechne Wydawnictwo Rolnicze i Leśne. Oddział, cop. Poznań, Polska.

Kucuk, C., and M. Kivanc. 2008. Mycoparasitism in the biological control of Gibberella zeae and Aspergillus ustus by Trichoderma harzianum strains. J. Agri. Technol. 4:49-55.

Kunitz, M. 1946. Spectrophotometric method for the measurement of ribonuclease activity. J. Biol. Chem. 164:563-568. 
Kurzatkowski, W., A. Törrönen, J. Filipek, R. Mach, P. Herzog, S. Sowka, and C.P. Kubicek. 1996. Glucose-induced secretion of Trichoderma reesei Xylanases. Appl. Environ. Microbiol. 62(8):2859-2865.

Larkin, M.A., G. Blackshields, N.P. Brown, R. Chenna, P.A. McGettigan, H. McWilliam, et al. 2007. ClustalW and ClustalX version 2 . Bioinformatics 23(21):2947-2948.

Latorre, B.A., E. Agosin, R. San Martín, y G.S. Vasquez. 1997. Effectiveness of conidia of Trichoderma harzianum produced by liquid fermentation against Botrytis bunch rot table grape in Chile. Crop Protection 16:209-214.

López-Pérez, P.A., M.I. Neria-González, R. Aguilar-López. 2013. Cadmium concentration stabilization in a continuous sulfate reducing bioreactor via sulfide concentration control. Chem. Pap. 67(3):326-335.

Lu, F., M. Lu, Z. Lu, X. Bie, H. Zhao, and Y. Wang. 2008. Purification and characterization of xylanase from Aspergillus ficuum AF-98. Bioresource Technol. 99:5938-5941.

Manocha, M.S., and A.S. Sahai. 1993. Mechanisms of recognition in necrotrophic and biotrophic mycoparasites. Can. J. Microbiol. 39:269-275.

Miller, G.L. 1959. Use of dinitrinosalicylic acid reagente for determination of reducing sugar. Anal. Chem. 31:426-428.

Mokhtar, H., and A. Dehimat. 2012. Antagonism capability in vitro of Trichoderma harzianum against some pathogenic fungi. Agric. Biol. J.N. Am. 3(11):452-460.

Monreal, J., and E.T. Reese. 1969. The chitinase of Serratia marcescens. Can. J. Microbiol. 15:689696.

Nawani, N., S.D. Nirpjit, and K. Jagdeep. 1998. A novel thermostable lipase from a thermophilic Bacillus sp. Characterization and esterification studies. Biotechnol. Lett. 20(10):9971000.

Nobe, R., Y. Sakakibara, N. Fukuda, N. Yoshida, K. Ogawa, and M. Suijo. 2003. Purification and characterization of laminaran hydrolases from Trichoderma viride. Biosci. Biotechnol. Biochem. 67:1349-1357.

Orole, O.O., and T.O. Adejumo. 2009. Activity of fungal endophytes against four maize wilt pathogens. Afr. J. Microbiol. Res. 3(12):969973.

Phillips-Mora, W., A. Coutiño, C.F. Ortiz, A.P. López, J. Hernández, and M.C. Aime. 2006. First report of Moniliophthora roreri causing frosty pod rot (moniliasis disease) of cacao in Mexico. Plant Pathol. 55:584.
PhyloDraw. 1999. PhyloDraw versión 0.8. Graphics Application Lab, Pusan National University. Available at http://search.wareseeker. com/phylodraw-version-0.8/ (Accessed 30 July 2008).

Quintana-Obregón, E.A., M. Plascencia-Jatomea, J. López-Cervantes, D.L.A. Cira-Chávez, D.I. Sánchez-Machado, and M.O. Cortez-Rocha. 2011. Antifungal activity of chitosan in Cladosporium cladosporioides isolated from safflower. Rev. Mex. Micol. 34:93-96.

Rawashdeh, R., I. Saadoun, and A. Mahasneh. 2005. Effect of cultural conditions on xylanase production by Streptomyces sp. (strain $\mathrm{Ib} 24 \mathrm{D})$ and its potential to utilize tomato pomace. Afr. J. Biotechnol. 4(3):251-255.

Rezende, M.I., A.M. Barbosa, A.F.D. Vasconcelos, and A.S. Endo. 2002. Xylanase production by Trichoderma harzianum rifai by solid state fermentation on sugarcane bagasse. Braz. J. Microbiol. 33:67-72.

Riascos, D., I. Quiroga, y L. Hoyos-Carvajal. 2011. Análisis de la sintomatología de la roña en gulupa (Pasiflora edulis f. edulis SIMS). Agron. 19(1):20-30.

Robeson, D.J., and M.A.F. Jalal. 1992. Formation of Ent-isophleichrome by Cladosporium herbarum isolated from sugar beet. Biosci. Biotech. Biochem. 56(6):949-952.

Schubert, K., J.Z. Groenewald, U. Braun, J. Dijksterhuis, M.S. Starink, C.F. Hill, et al. 2007. Biodiversity in the Cladosporium herbarum complex (Davidiellaceae, Capnodiales), with standardisation of methods for Cladosporium taxonomy and diagnostics. Studies in Mycology 58:105-156.

Seiboth, B., L. Hartl, M. Pail, and P.C. Kubicek. 2003. D-Xylose metabolism in Hypocrea jecorina; loss of the xylitol dehydrogenase step can be partially compensated for by lad1-Encodad L-Arabinitol-4-Dehydrogenase. Eukaryot. Cell. 5:867-875.

Stricker, R.A., K. Grosstessner-Hain, E. Wurleitner, and P.L. Mach. 2006. Xyr1 (Xylanase Regulator 1) Regulates both the hydrolytic enzyme system and D-Xylose metabolism in Hypocrea jecorina. Eukariot. Cell. 12:2128-2137.

Suarez, M.B., L. Sanz, M.L. Chamorro, M. Rey, F.J. Gonzalez, A. Llobell, and E. Monte. 2005. Proteomic analysis of secreted proteins from Trichoderma harzianum. Identification of a fungal cell wall-induced aspartic protease. Fungal Genet. Biol. 42:924-934.

Szekeres, A., B. Leitgeb, L. Kredics, L. Manczinger, and C. Vágvölgyi. 2006. A novel, image analysis-based method for the evaluation of in vitro antagonism. J. Microbiol. Methods 65(3):619-622. 
Ujiie, M., C. Roy, and M. Yaguch. 1991. Low-molecular weight xylanase from Trichoderma viride. Appl. Environ. Microbiol. 57(6):18601862.

Velázquez-del Valle, M.G., S. Bautista-Baños, A.N. Hernández-Lauzardo, M.G. Guerra-Sánchez, and E. Amora-Lazcano. 2008. Estrategias de control de Rhizopus stolonifer Ehrenb. (EX FR) Lind, agente causal de pudriciones postcosecha en productos agrícolas. Rev. Mex. Fitopatol. 26(1):49-55.

Villadsen, J., J. Nielsen, and G. Lidén. 2011. Bioreactor engineering principles. 3r. ed. Springer, USA.

Wang, H.C., J. Wang, W.H. Li, Y.F. Huang, H.Q. Xia, M.S. Wang, et al. 2014. Cladosporium cladosporioides identified in China on tobacco seeds. Plant Dis. 98(7):1002. 